\title{
Systemic non-albicans infections presented as meningitis in chronic hepatitis B patient: a case report
}

\author{
Wen-Jing Lv, Hui Bu, Jun-Ying He, Ran-Ran Sun, Yue-Li Zou \\ Department of Neurology, The Second Hospital of Hebei Medical University, Shijiazhuang 050000, Hebei, China.
}

\section{A B S T R A C T}

Non-albicans candida meningitis is a relatively rare disease, with nonspecific clinical manifestation, which makes the misdiagnosis occur sometimes, especially in the early stage of the disease. Abuse of broad-spectrum antibiotics, corticosteroids, central vein cannulas, senility, big operation, malignancy, and total parenteral alimentation were all the susceptible factors of non-albicans candida infection. We present a case of this type of non-albicans infection in a 42-year-old woman who was early misdiagnosed as tuberculous meningitis and was treated with antibiotics and antituberculosis agents. The diagnosis of non-albicans infection was confirmed by fungus culture of the cerebrospinal fluid (CSF) with a low detectable rate. This case reminds us that the non-albicans candida meningitis had a nonspecific clinical presentations and laboratory data, and was difficult to differentiate from tuberculosis meningitis. Hence, we should highly suspect this disease if central nervous system infections with uncertain pathogens. Test cell counts; protein and fungus culture of CSF should be used to confirm the diagnosis. Once the diagnosis was established, the patients should receive antifungal treatment based on drug sensitivity tests as early as possible.

Key words: Central nervous system, fungi, non-albicans candida

\section{INTRODUCTION}

Central nervous system (CNS) infection caused by candida is a type of systemic candidiasis ${ }^{[1]}$ It is rare in clinical practice, with unnspecific clinical features and laboratory data, which makes this disorder prone to misdiagnosis. The morbidity of non-albicans candida infection rises in recent years as the abuse of broad-spectrum antibiotics and corticosteroids, human immunodeficiency virus (HIV) infections, and so on. Here, we present candidal meningitis case mimics tuberculous meningitis in a chronic hepatitis B patient.

\section{CASE REPORT}

A 42-year-old female, farmer was admitted in the Neurology Department of our hospital on November $24^{\text {th }}$, 2009. Two months earlier, she had a sharp, intermittent occipital headache and fever, the temperature fluctuated

\begin{tabular}{|l|l|}
\hline \multicolumn{2}{|c|}{ Access this article online } \\
\hline Quick Response Code: & \\
\hline & Website: \\
\hline & Www.nnjournal.net \\
\cline { 2 - 3 } & \\
\hline
\end{tabular}

between $37.5^{\circ} \mathrm{C}$ and $38.5^{\circ} \mathrm{C}$ and up to $40^{\circ} \mathrm{C}$ sometimes, it increased at dusk and night, accompanied with nonprojectile vomiting occasionally and anorexia with no visual blurring, diplopia, preceding trauma, or history of migraine. The fever and headache continued for 2 months. Painless intumescent lymph nodes showed up in the neck 2 months later, and the patient was diagnosed as tuberculous infection. And treated with antibiotics and antituberculosis drugs in local hospitals, but the symptoms were not relieved. So she came to our hospital for further diagnosis and treatments. Physical examination revealed an ill-looking woman with yellow skin, white conjunctiva, and enlarged cervical lymph nodes. The neurological examination showed neck stiffness, positive Kernig's sign, and negative Babinski sign on both sides.

She received lumber puncture in the local hospital, the pressure of cerebrospinal fluid (CSF) was $265 \mathrm{mmH}_{2} \mathrm{O}$, with normal cell counts, glucose, chlorides, and protein. The white blood cell account was $10.6 \times 10^{9} \mathrm{~L}$, the neutrophilic granulocyte percentage was $72.9 \%$. The erythrocyte sedimentation rate was $76 \mathrm{~mm} / \mathrm{h}$, liver function tests showed total bilirubin $75 \mu \mathrm{mol} / \mathrm{L}$, direct bilirubin $40.7 \mu \mathrm{mol} / \mathrm{L}$, indirect bilirubin 34.3 $\mu \mathrm{mol} / \mathrm{L}$, aspartate aminotransferase $89 \mathrm{U} / \mathrm{L}$, alanine aminotransferase $67 \mathrm{U} / \mathrm{L}$, alkaline phosphatase 323 $\mathrm{U} / \mathrm{L}$, and $\gamma$-glutamyltransferase $400 \mathrm{U} / \mathrm{L}$. Serology for 
HIV was negative, the hepatitis B surface antibody, hepatitis B core antibody, hepatitis B e antibody were all positive. Lung computed tomography (CT) scans showed nodules and fibrous stripes in the right middle lobe, left apex, and lingular lobe, with multiple enlarged lymph nodes in the mediastinum. We repeated a lumbar puncture on November $16^{\text {th }}, 2009$. The CSF tests showed: pressure $204 \mathrm{mmH}_{2} \mathrm{O}$, white blood cell count $36 \times 10^{6 /} \mathrm{L}$, protein $0.48 \mathrm{~g} / \mathrm{L}$, glucose $2.70 \mathrm{mmol} / \mathrm{L}$, and chloride $117 \mathrm{mmol} / \mathrm{L}$. The CSF cytology showed that lymphocytes increased mainly. Both acid-fast staining and the antigen of the tubercle bacillus were negative. Brain magnetic resonance imaging (MRI) scans [Figure 1] presented multiple high signal lesions on T2-weighted imaging (T2-WI) and fluid attenuated inversion recovery (FLAIR) (T2-WI and FLAIR). Remarkable intensify leptomeninges and abnormal strengthening signal in the right side of the caudate nucleus were observed. It was necessary to rule out metastatic tumor or granulomatous according to the history. Hence, we tested the carcinoembryonie

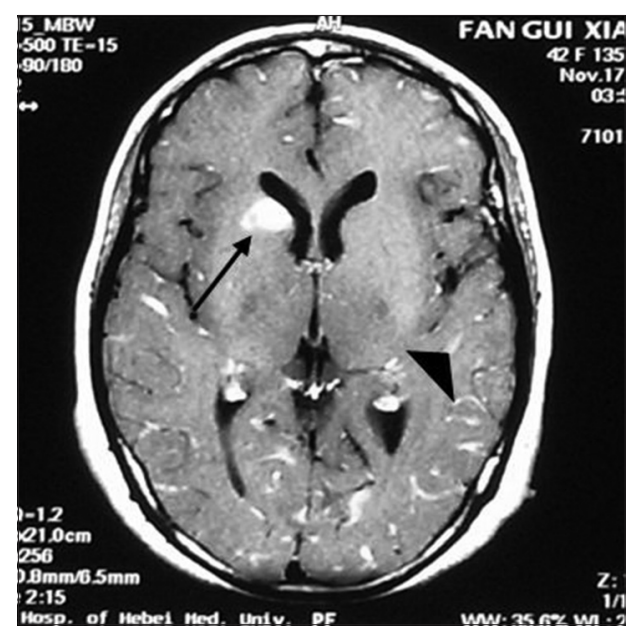

Figure 1: Brain magnetic resonance imaging scan presented multiple high-signal lesions on T2-weighted and fluid attenuated inversion recovery. Remarkable intensity of leptomeninges and abnormal strengthening in the right side of the caudate nucleus

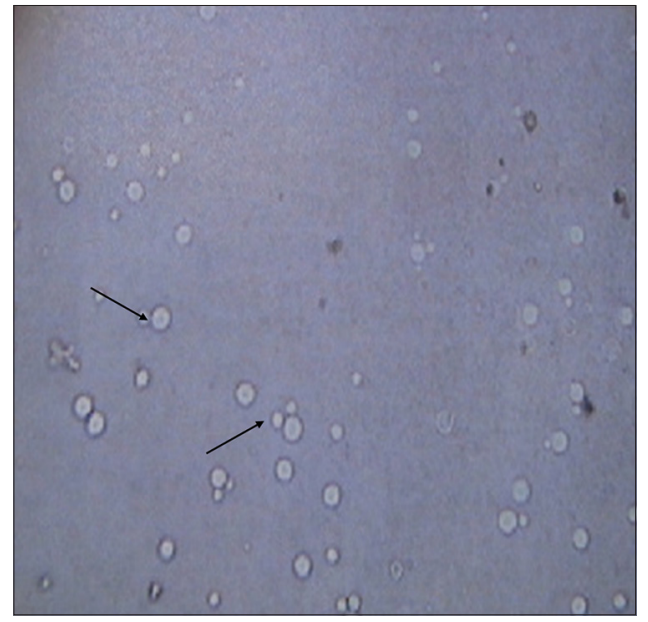

Figure 3: Cerebrospinal fluid and lymph node puncture fluid culture showed positive identification of blastoconidia (arrow) with India ink staining, but was not observed capsules antigen, alpha fetal protein, and carbohydrate antigens (CA-199, CA-125) in the blood. Only CA-199 was moderately elevated $(90.1 \mathrm{U} / \mathrm{mL})$. B-mode ultrasound scans of the abdomen revealed multiple low signals in the hepatic hilar region and splenic hilum region. Ultrasound doctor considered them as enlarged lymph nodes. CT intensified scans of the upper abdomen showed an occupied lesion in the right hepatic lobe with intra-hepatic bile duct dilation. Radiologists thought the occupied lesion was cholangiocarcinoma, combining with the history we thought it was apt to inflammatory pseudotumor, but could not exclude malignant tumor, however, because systemic candidiasis is always seen in immunocompromised individuals.

Cervical lymph node biopsy [Figure 2] showed mycotic lymphadenitis. There were a lot of granulomatosis-like structures and mold in macrophages. Lymph node puncture fluid smears [Figure 3] showed that hyphae were visible. CSF smears showed no fungus. CSF and lymph node puncture fluid cultures [Figure 4] we saw blastoconidia with India ink staining, and not saw capsule in the culture. Non-albicans candida was

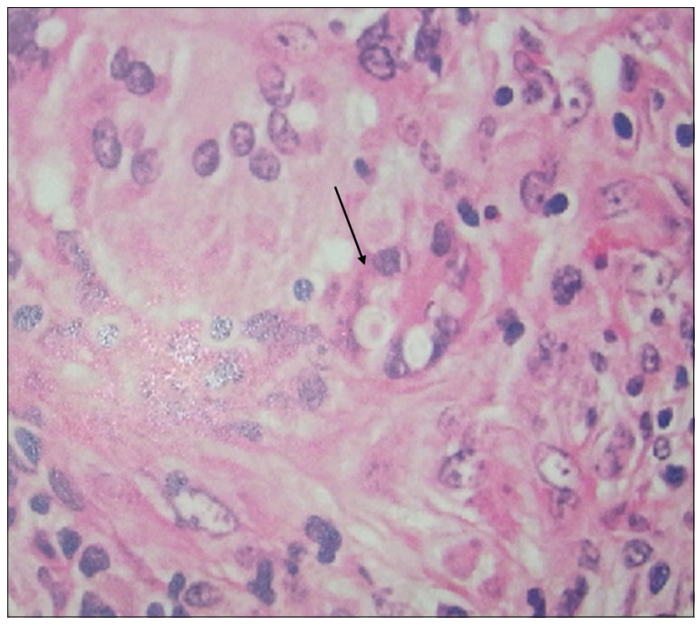

Figure 2: Cervical lymph node biopsy showed mycotic lymphadenitis (arrow). There were a lot of granulomatosis-like structures and mold in macrophages

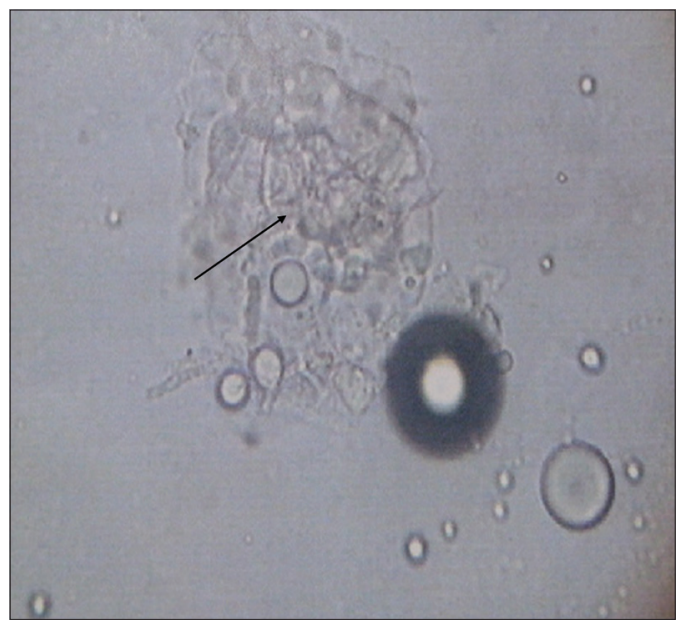

Figure 4: Lymph node puncture fluid smears showed that hyphae (arrow) was visiable 
considered as a pathogen, and the diagnosis was clear as systemic non-albicans candida infections mainly presented as non-albicans candidal meningitis. She received amphotericin B $1 \mathrm{mg} /$ day and fluconazole $400 \mathrm{mg} /$ day intravenously as soon as diagnosis is clear. Unfortunately, the patient discharged from the hospital on the second day since administration of antifungal drugs because of economic reasons and hence we could not observe the therapeutic effect.

\section{DISCUSSION}

Many strains of fungal can cause CNS infection. Aspergillus, mucor, cryptococcosis, and yeast are the most common strains in China. ${ }^{[2]} \mathrm{A}$ foreign research showed that yeast, aspergillus, and cryptococcosis was the most common fungal pathogens for CNS infection in immune-competent hosts, while aspergillus, candida and nocardia were the most common pathogens in patients with impaired immunity. ${ }^{[3-5]}$ Many factors contributed to the increasing incidence of systemic candidiasis, such as HIV transmission, the using of hormonal, immunosuppressive agents and broad-spectrum antibiotics, organ transplantation, the invasive intracranial examination, and so on. This patient has an infection of hepatitis B virus, and uncertained cholangio carcinoma. Her immune system was weak, such kind of patients should be considered of rare bacterial or fungus infection at the initial of treatment. Therefore, fungi, and bacterial culture could be done more actively.

Candida albicans was the most common pathogen of candidiasis in the past, ${ }^{[6]}$ and the proportion of nonblastomyces albicans infection has tended to rise recently. However, meningitis caused by non-albicans candida is rare in the clinic and does not have specific manifestations, which make the misdiagnosis occur sometimes, especially in the early stage of the disease. This case reminds us that candidiasis should be considered if we found patients had unexplained fever responded poorly to antibiotics, thrush, esophagitis, vaginitis, atypical lung infiltrates, unexplained liver dysfunction, mental abnormality, endoophthalmitis, dry cough, rash, and tender muscle. ${ }^{[7]}$ Candida is a kind of mold and belongs to Saccharomyces. It includes two types-yeast type and pseudohyphae type. Yeast type was round or oval and can produce blastoconidia. Monilia albicans, candida parapsilosis, candida tropicalis, candida glabrata, monilia guilliermondii, candida lusitaniae, and candida krusei are the most common candida albicans that can infect humans. In America and other European countries, candida parapsilosis and candida krusei are the major pathogens of non-albicans candida infection. ${ }^{[8]}$ These fungus are resistant to the most common antifungal drugs, which makes them more likely to be opportunistic infections. The situation is more or less similar in China and Brazil..9]

We summarize, the clinical features of this patient: (1) a 42-year-old woman, acute onset of fever, which is resistant to the antibiotics and antituberculosis agents; (2) liver dysfunction, intra-liver lesion, biliary ducts dilation; (3) hilar and portal hepatis lymph nodes enlargement; (4) used of broad-spectrum antibiotics in the early stage of the disease when the diagnosis was unclear; (5) with a low immune state, which was the basis of opportunistic infections. We considered the case was blastomycosis according to the lymph node biopsy results. Morphologically, giant blastoconidia presented a spectrum of forms such as blastoconidia with linear creases, with single broad-based buds resembling Blastomyces dermatididis, with multiple buds resembling Paracoccidioides brasiliensis. Its forms varied along with the environment and temperature. Such as, after growth on commercially prepared cultures in room temperature, we can see white fluffy colonmold to naked eyes and the characteristic thick-walled broad-based yeast in microscope with Periodic acid Schiff staining, as it shows mycelia-like type, while been cultured in $37^{\circ} \mathrm{C}$ it presents on brown and frilly yeast-like colony, and in tissue it shows yeast-like type. Candida includes yeast type and pseudohyphae type and produces blastoconidium. In this case, we can see blastoconidia only with India ink staining in CSF and lymph node puncture fluid culture. Brain biopsy hadn’t been done. Combing with the manifestation, brain MRI, CSF tests, and fungus culture results, we highly suspected systematic candidal albicans infection. This case reminds us that it is essential to do CSF culture for patients with unexplained CNS infection, not only the routine CSF cell counts and protein tests. If the diagnosis is established, patients should receive antifungal treatment based on drug sensitivity test as early as possible.

\section{REFERENCES}

1. Liu Q, Lv XJ. The advances of central nervous system fungal infections. West China Med J 2007;22:654-7.

2. Wang JM, Chen QX, Tian DF. Craniocerebral fungal infection in neurosurgery (analysis of 14 cases). Chin J Clin Neurosurg 2006;11:70-3.

3. Yamada K, Zoarski GH, Rothman MI, Zagardo MT, Nishimura T, Sun CC. An intracranial aspergilloma with low signal on T2-weighted images corresponding to iron accumulation. Neuroradiology 2001;43:559-61.

4. Kopp W, Fotter R, Steiner H, Beaufort F, Stammberger H. Aspergillosis of the paranasal sinuses. Radiology 1985;156:715-6.

5. Tempkin AD, Sobonya RE, Seeger JF, Oh ES. Cerebral aspergillosis: radiologic and pathologic findings. Radiographics 2006;26:1239-42.

6. Xu YC, Wang P, Chen MJ. Pfizer global multicenter yeast sensitivity monitoring results. Chin J Infect Chemother 2003;3:187-90.

7. Chen HD. The diagnosis and treatment of systemic candidal infection. J Intern Intensive Med 2005;11:19-20. 
8. Perez-Nadales E, Almeida Nogueira MF, Baldin C, Castanheira S, El Ghalid M, Grund E, Lengeler K, Marchegiani E, Mehrotra PV, Moretti M, Naik V, Oses-Ruiz M, Oskarsson T, Schäfer K, Wasserstrom L, Brakhage AA, Gow NA, Kahmann R, Lebrun MH, Perez-Martin J, Di Pietro A, Talbot NJ, Toquin V, Walther A, Wendland J. Fungal model systems and the elucidation of pathogenicity determinants. Fungal Genet Biol 2014;70: 42-67.
9. Colombo AL. Epidemiology and treatment of hematogenous candidiasis: a Brazilian perspective. Braz J Infect Dis 2000;4:113-8.

Cite this article as: Lv WJ, Bu H, He JY, Sun RR, Zou YL. Systemic nonalbicans infections presented as meningitis in chronic hepatitis $\mathrm{B}$ patient: a case report. Neuroimmunol Neuroinflammation 2014;1(3):161-4.

Source of Support: Nil. Conflict of Interest: No.

Received: 19-06-2014; Accepted: 01-09-2014 